\title{
Angebot für Mitglieder des FVDZ: Maßgeschneiderte Praxis suchen - und finden lassen
}

\begin{abstract}
Für jeden Topf gibt es einen passenden Deckel, lautet ein altes Sprichwort. In Liebesdingen ist damit gemeint, dass jeder schon irgendwann den geeigneten Partner findet. Was fürs Privatleben stimmt, kann im Berufsleben nicht schlecht sein. Im Praxispool des Freien Verbands Deutscher Zahnärzte gibt es jede Menge Töpfe für alle möglichen Deckel. Wer eine Praxis zur Übernahme sucht, wird einen Abgeber finden und umgekehrt. Das Praktische für Abgeber und Übernehmer gleichermaßen: Der FVDZ-Praxispool trifft eine Vorauswahl und findet Partner, die zueinander passen.
\end{abstract}

$\mathrm{U}$ nd so funktioniert der Praxispool: Sowohl Praxisübernehmer als auch -abgeber finden im mitgliedergeschützten Bereich unter www.fvdz.de recht detaillierte Fragebögen. Im Fragebogen für Übernehmer werden Daten zur „Wunschpraxis“ abgefragt (beispielsweise Praxisform, Tätigkeitsschwerpunkte, aber auch zu den Räumlichkeiten und der Ausstattung). Im Fragebogen für Abgeber wird gefragt, was die Praxis zu bieten hat.

Das Besondere: Die Daten werden nicht, wie bei Partnerportalen oder anderen Praxisbörsen, veröffentlicht, sondern von einer Mitarbeiterin des FVDZ gesammelt und ausgewertet, um dann geeignete Interessenten gezielt zusammenzuführen. So hat jedes FVDZ-Mitglied die Möglichkeit, quasi einen eigenen Agenten zu nutzen, um in eine geeignete Praxis einzusteigen oder eine Praxis zu übernehmen, die örtlich bereits etabliert ist. Die lästige Recherche entfällt.

Die Vermittlung erfolgt selbstverständlich neutral, diskret und kostenfrei. Weitere Fragen beantwortet Frauke Garstka in der FVDZ-Bundesgeschäftsstelle in Bonn gern unter der Telefonnummer 0228 / 855732 oder per E-Mail unter praxis-pool@ fvdz.de.

\section{FVDZ - kurz gemeldet}

\section{Frag den FVDZ!}

Unter dem Motto „Frag den FVDZ“ geben ausgewählte Referenten der FVDZ-Akademie Expertentipps rund um die Zahnarztpraxis auf dem YouTube-Kanal des Freien Verbands : Deutscher Zahnärzte (Stichwort FVDZ Presse). Die Referenten beantworten in kurzen kostenlosen Videoclips Fragen zu Themen wie „Controlling in der Zahnarztpraxis“ oder „Mein erster Job als Zahnarzt“. In dem aktuellen Online-Expertentipp informiert Rechtsanwalt Michael Lennartz über das Thema „Der Praxismietvertrag - das übersehene Problem?“

\section{Jetzt anmelden: Zahnärzte-Sommerkongress auf Usedom}

Der FVDZ lädt Zahnärzte und ihr Praxisteam vom 23. bis 27. Mai zur traditionellen Fortbildungswoche in Heringsdorf : auf der Insel Usedom ein. Der Kongress steht für neueste wissenschaftliche Erkenntnisse und Behandlungsmethoden in der Zahnmedizin. Zum Programm zählen unter anderem Referate rund um die Zahnerhaltung sowie zur plastischen Parodontalund Implantatchirurgie. Auch rechtliche und betriebswirtschaftliche Tipps kommen nicht zu kurz. Am Abschlusstag: blickt Redner und Coach Ralph Goldschmidt in seinem Vortrag "Shake your Life!" über den Tellerrand der Zahnarztpraxis und gibt Tipps für ein zufriedenes und erfolgreiches Leben. Die Teilnahme steht FVDZ-Mitgliedern wie Nicht-Mitgliedern offen. Weitere Infos auf der Verbandswebsite unter der Rubrik "FVDZ akademie", oder bei Corrine Trommeschläger, ct@fvdz.de, Tel. 0228 8557-34.

\section{Hier steht eine Anzeige.}

